\title{
SYSTEMS OF THE INFORMATION SOCIETY
}

\author{
Professor Simon Rogerson \\ Centre for Computing and Social Responsibility \\ De Montfort University \\ The Gateway \\ Leicester \\ LE1 9BH \\ UK \\ srog@dmu.ac.uk
}

\begin{abstract}
An inclusive information sociery needs an infrastnucture built upon systems which are in harmony with citizens. This paper discusses some of the contextual issues which need to be addressed to realise this goal. There is a need to strive continually to develop adaptive systems which satisfy the dynamic needs of people. The project management of such development activities must be sensitive to the wider social issues. The development activity must adopt a sociotechnical perspective. It must never be forgotten how vulnerable individuals and society are from insecure and inappropriate information systems. It must be recognised that the social systems that are dependant on information systems to survive can be very unpredictable in terms of the benefits gained and costs incurred by both individuals and society at large.
\end{abstract}

\section{INTRODUCTION}

The computing technology revolution continues to accelerate. The applications of this universal tool seem limitless. Indeed the convergence of computing, media and communication technologies has meant that each day we become more and more dependant on technology at work, at home, in travel, in learning and in communicating. Ubiquitous computing has moved from the backroom to everyday applications. Thus systems based on computing technology are powerful change agents. We need to think carefully about the ramifications of our actions. We need to consider not only the technological and economic issues but also the ethical and social issues when developing and implementing such systems.

This special edition of the Australian Journal of Information Systems is based on a selection of papers presented at ETHICOMP 2001 held at the Technical University of Gdansk, Poland in June 2001. The overall theme of the conference was "Systems of the Information Society". The aim was to focus on the ethical and social impacts of these systems on society, organisations and individuals. This was done from four perspectives: software engineering and systems development; teaching ethics to computing students; ethics in virtual communities; and ethics in the off-line world. The papers included in this edition of the journal reflect many of these themes and illustrate some of the major issues that need to be addressed.

\section{SYSTEMS IN CONTEXT}

Information systems are built by people to be used by people for the benefit of people. The development process is a human activity system and the resulting information systems are used in another human activity systems. A human activity system (HAS) is defined by Banathy (2000) as, "an assembly of people and other resources organized into a whole in order to accomplish a purpose. The people in the system are affected by being in the system, and by their participation in the system they affect the system. People in the system select and carry out activities -- individually and collectively -- that will enable them to attain a collectively identified purpose."

To realise acceptable information systems the development HAS must be founded on some key concepts as shown in Figure 1. There are two elements of development; process and management. The process must focus on an effective and sensitive build whilst management must be empowering in order to realise the building process. Those involved in the development must represent all stakeholders. It is essential to adopt a wider perspective which includes those directly and indirectly affected by the system to be developed. This broadening is essential as it includes not only traditional stakeholders viewed from the widely-accepted business perspective but also social actors who Schot (2001) defines as, "those who experience the effects of evolving technologies but [traditionally] are not actively involved in the development of technologies. They can be consumers, citizens, employees, companies, social groups etc." 


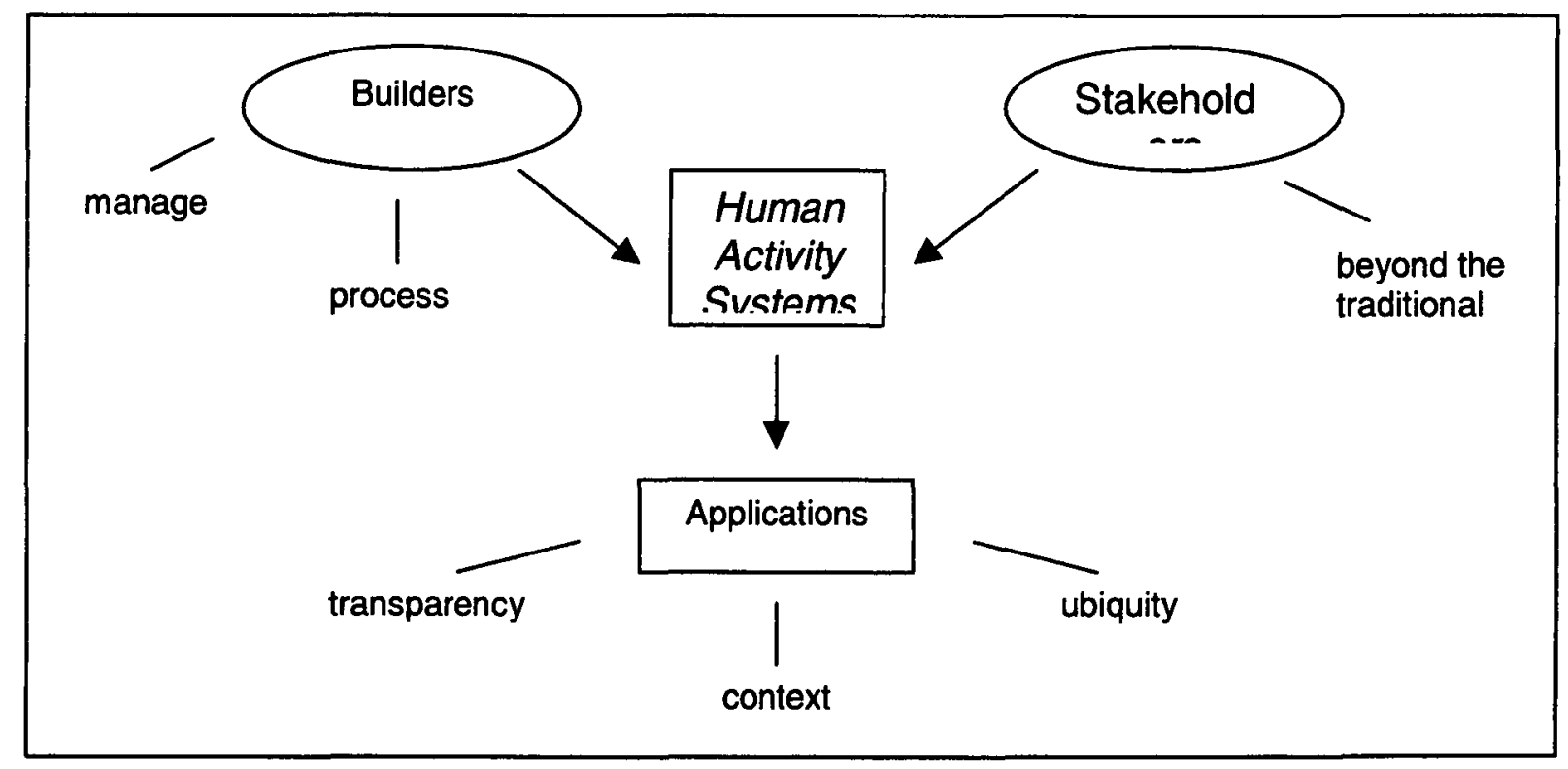

Figure 1 Systems in context

Systems need to be viewed from the perspective of everyday applications. Ubiquitous computing exists primarily because the majority of everyday activities can be defined as a series of inputs, processes and outputs. As the computer technologies advance and applications become commonplace so transparency increases. People do not give the technology a second thought. It becomes invisible and only the system outcomes are noticed. The settings of applications vary considerably because of differences in culture, religious faith, age, gender, socioeconomic status, literacy levels and technical literacy levels. Thus the context of systems demands a broad consideration of the issues. It is a move from a blinkered technological approach to an enlightened inclusive approach.

\section{Barriers and challenges}

A number of barriers and challenges exist for the policy makers, managers, developers and implementers of systems. There is a tendency to overlook considerations other than the traditional technical and economic ones. As society's dependency on technology increases the risk increases of creating dangerous systems. Technological dependency has a tendency to limit choice implicitly or explicitly as resource availability reduces for maintaining non-technological facilities. However, it will be several generations before the digital solution is widely accepted as the traditional and obvious solution. Acceptance is affected by whether a sense of ownership of systems exists within the using community. Such challenges point to the need on the part of the builders to accept their social responsibility and act in a way that attacks the digital divide.

\section{The digital divide}

According to the report by Booz Allen and Hamilton (2000) Britain is not leading in terms of Internet penetration. Figure 2 shows a three tier situation. In the US, six out of ten people regularly access the Internet from work, home or a public access terminal. In Scandinavia around $40 \%$ do so. A noticeable gap currently exists between the world's most connected nations in a first tier, and a second tier of nations with penetration levels in the range $15-25 \%$. The UK is top of the second tier. The third tier shows those lagging behind. But even these are way ahead of a much larger group of countries. 


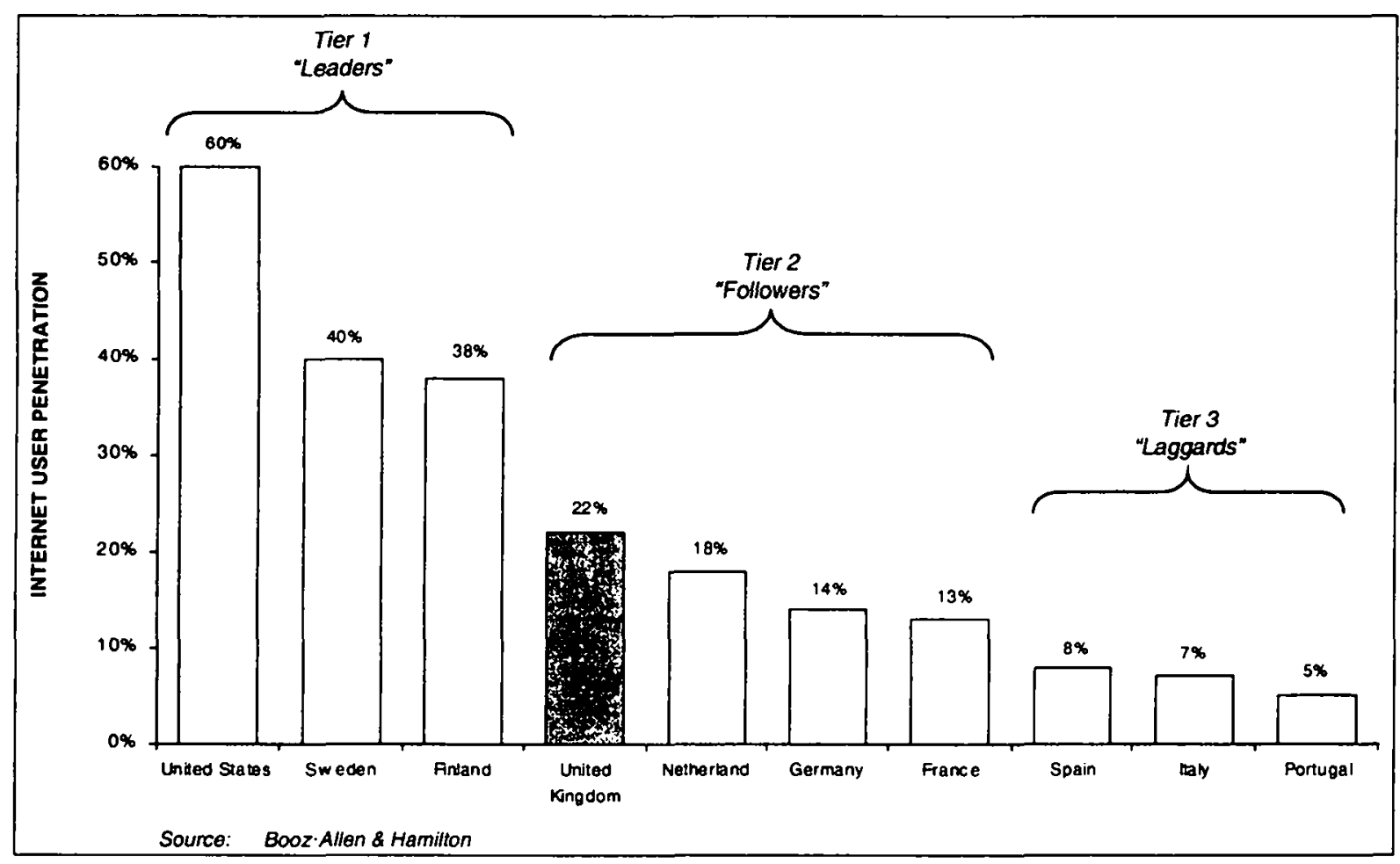

Figure 2 Internet penetration

The consensus of the plethora of statistics is that only about $7 \%$ to $10 \%$ of the world's population is on-line. In global terms the dichotomy of "haves" and "have nots" is vast. With the potential benefit to society and its citizens this virtual divide should be a cause for great concern. Consider some of the facts. According to the US Internet Council's State of the Internet Report for the year 2000, there is considerable disparity within the continent of Africa in terms of Internet accessibility. Africa has more than $10 \%$ of the world's population but less than $1 \%$ of the world's Internet users. A million South Africans have access to the Web, but practically nobody does in the Congo. Looking at South Africa in more detail, a 1999 survey of 2000 black South African men and 4000 black South African women by Webcheck revealed minimal Internet access rates among black Africans. Out of those surveyed only two men and five women had Web access at home and only 24 men and 24 women had Web access at work. Turning to India, according to eMarketer (autumn 2000), the number of active adult Internet users in India was 270,000 at the end of 1999 and was set to reach 1.5 million by the end of 2000 , but this is only $0.2 \%$ of India's adult population. eMarketer attributes the low Internet penetration in India to extreme and widespread poverty, compounded by a poor telecommunications infrastructure and limited PC ownership.

\section{Citizen education}

Does education have a role to play in reducing the digital divide? Consider the example of electronic commerce. In order to participate fully people need increasing Internet skills and knowledge as illustrated in the diagram by Booz Allen and Hamilton (2001) in Figure 3. 


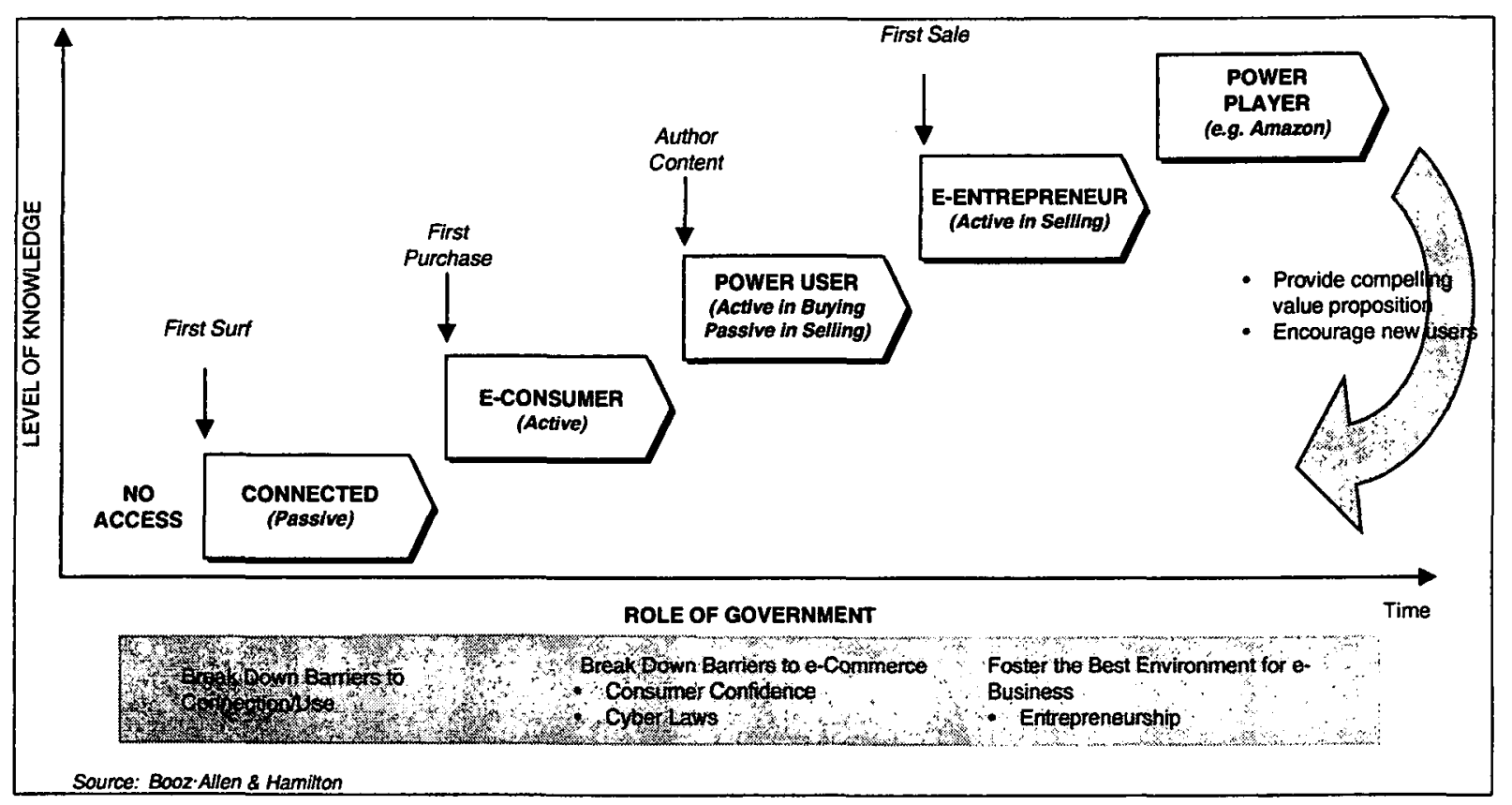

Figure 3 Increasing Internet skills

In their report Booz Allen Hamiliton explain that, "To fully capture the benefits of the Knowledge Economy, users need more than mere availability, and must move beyond passive browsing. They must become active users - participating in discussions, educating themselves, making transactions and engaging in commerce. At the next level, the Internet offers unprecedented opportunity for consumers to become entrepreneurs themselves; setting up web sites, and ultimately even businesses [see Figure 3]. Government can play a role not simply in getting people online, but in creating conditions and incentives for them to evolve into active users - even 'power users'." In other words through education ordinary people can become more and more proactive to the point where they could be a major player in dictating the nature and influencing the level of penetration of electronic commerce.

\section{Policy and politics}

The direction of policy and political strategy has a significant impact on the way systems are perceived and used in the information society. Johnson (2000) suggests that there should be a continual assessment of the Internet which is the backbone of the information society. This assessment should focus on societal worth and be based on the following questions:

- How is the Internet governed or should it be?

- How is autonomy of nations (states or local communities) recognised in policy decisions regarding the Internet or how should it be?

- Are the economic interests shaping the Internet allowed to undermine its potential to serve political and public interests or should it be?

- Is freedom of expression being protected or should it be?

- To what extent is there privacy on the Internet and to what extent is the medium a medium of surveillance? (Johnson, 2000)

Whether such assessment takes place and whether it is effective depends to some extent on political thrust in the major nations of the information society (these nations could reasonably be defined according to Figure 2 above). There are two dimensions to this thrust which appear significant. Is an apocalyptic approach adopted which scraps all existing systems in favour of virtual alternatives. This is in contrast to an evolutionary approach which replaces traditional systems with virtual alternatives where appropriate and has long transition periods to promote acceptance. The second dimension is concerned with computer technology as a driver. There are those who firmly believe that computer technology and its ability to create a virtual world is the only way to move forward. Such "wired-up thinking" is badly flawed. What is required is "joined-up thinking" where the technology is viewed as one of several options available to resolve problems and create new opportunities for social benefit and wealth creation. 


\section{CONCLUSION}

It seems clear from the issues raised so far that an inclusive information society needs an infrastructure built upon systems which are in harmony with citizens. The papers that follow illustrate this point. Barbara Bergier argues that human-centred goals must be a priority when considering the development and implementation of systems. In this way we are more likely to get systems that are adaptive to our needs rather than systems that demand us to be adaptive to the foibles of poor system design. Don Gotterbarn argues that narrow risk assessment and a narrow scope of software significantly contribute to system failures. He presents a new project management approach in order to encourage a wider consideration and thereby reduce the likelihood of system failures. Joe Thomas and Andy Bissett discuss a case study about an assessment and referral process for drug misusers. They highlight the serious problems that can arise through using an information system as part of this process. Paula Roberts and Jenny Webber discuss the role of computer hackers in society. They take a fresh look at how we might limit damage by malicious actions. Finally, Helen Richardson and Kate Richardson describe a case study of a call centre and the use of a customer relationship management system. They discuss the contrasting opinions as to the worth of this type of human activity system.

There are some important messages in this collection of papers. We must continually strive to develop adaptive systems which satisfy the dynamic needs of people. The project management of such development activities must be sensitive to the wider social issues. The development activity must adopt a socio-technical perspective. We must never forget how vulnerable individuals and society are from insecure and inappropriate information systems. Finally, the social systems that are dependant on information systems to survive can be very unpredictable in terms of the benefits gained and costs incurred by both individuals and society at large.

\section{REFERENCES}

Booz-Allen and Hamilton (2000) Achieving Universal Access, Booz-Allen and Hamilton, London, March. Johnson, D. G. (2000) Democratic Values and the Internet, in Langford, D. Internet Ethics, MacMillan Press, New York, Chapter 8, pp 181-199.

Schot J. (2001) Constructive technology assessment as reflexive technology politics, in Goujon, P., Dubreuil, B.H., (eds), Technology and Ethics: A European Quest for Responsible Engineering, Peeters, Leuven, Belgium, Chapter 2.1.1, pp 239-249. 